\title{
Existence and localization of positive solutions for a fractional boundary value problem at resonance
}

\author{
Samia Kouachi ${ }^{1 *}$, Assia Guezane-Lakoud ${ }^{1}$ and Fateh Ellagoune ${ }^{2}$
}

\section{"Correspondence:}

sa.kouachi@gmail.com

${ }^{1}$ Laboratory of Advanced Materials,

Faculty of Sciences, Badji

Mokhtar-Annaba University,

P.O. Box 12, Annaba, 23000, Algeria

\begin{abstract}
We investigate the existence of positive solutions for a fractional boundary value problem at resonance. By means of a fixed point theorem of increasing operators, the minimal and maximal nonnegative solutions for the problem are obtained.
\end{abstract}

MSC: 34B15; 34B18; 34A08; 26A33

Keywords: fractional boundary value problem; resonance; positive solutions; fixed point theorem

\section{Introduction}

We are concerned with the following fractional boundary value problem $(\mathrm{P})$ :

$$
\begin{aligned}
& { }^{c} D_{0^{+}}^{\alpha} u(t)=f(t, u(t)), \quad 0<t<1, \\
& u(0)=u^{\prime}(0)=0, \quad u^{\prime \prime}(0)=2 u(1),
\end{aligned}
$$

where ${ }^{c} D_{0^{+}}^{\alpha}$ denotes the Caputo fractional derivative, $2<\alpha<3$. We assume that $f:[0,1] \times$ $\mathbb{R}^{+} \rightarrow \mathbb{R}^{+}$is continuous. The boundary value problem (P) is said to be at resonance if the linear equation $L u={ }^{c} D_{0^{+}}^{\alpha} u(t)$ with the boundary value conditions (1.2) has a nontrivial solution, i.e., $\operatorname{dim} \operatorname{ker} L \geq 1$.

In recent years, there has been much work related to boundary value problems at resonance for ordinary or fractional differential equations. We refer the reader to [1-8] and the references therein. In most papers mentioned above, the coincidence degree theory was applied to establish existence theorems. In [9-11], the authors obtained the minimal and maximal positive solutions by using a fixed point theorem of increasing operators.

In this paper, we use this method to solve the boundary value problem (P). For the convenience of the reader, we recall some notations.

Let $X$ and $Y$ be real Banach spaces, $L: \operatorname{dom}(L) \subset X \rightarrow Y$ be a Fredholm operator of index zero. The map $N: X \rightarrow Y$ is called $L$-compact on $\bar{\Omega}$ if $Q N(\bar{\Omega})$ and $K_{p}(I-Q)(\bar{\Omega})$ are both compact.

Let $P: X \rightarrow X, Q: Y \rightarrow Y$ be continuous projectors such that $\operatorname{Im} P=\operatorname{Ker} L, \operatorname{Ker} Q=\operatorname{Im} L$ and $X=\operatorname{Ker} L \oplus \operatorname{Ker} P, Y=\operatorname{Im} L \oplus \operatorname{Im} Q$. It follows that $\left.L\right|_{\operatorname{Ker} P \cap \operatorname{dom}(L)}: \operatorname{Ker} P \cap \operatorname{dom}(L) \rightarrow$ $\operatorname{Im}(L)$ is invertible.

(c) 2015 Kouachi et al. This article is distributed under the terms of the Creative Commons Attribution 4.0 International License (http://creativecommons.org/licenses/by/4.0/), which permits unrestricted use, distribution, and reproduction in any medium, provided you give appropriate credit to the original author(s) and the source, provide a link to the Creative Commons license, and indicate if changes were made. 
We denote the inverse of $L$ by $K_{P}: \operatorname{Im} L \rightarrow \operatorname{Ker} P \cap \operatorname{dom}(L)$. Moreover, $\operatorname{since} \operatorname{dim} \operatorname{Im} Q=$ $\operatorname{dim} \operatorname{Ker} L<\infty$, there exists an isomorphism $J: \operatorname{Im} Q \rightarrow \operatorname{Ker} L=\operatorname{Im} P$. Set $H=L+J^{-1} P$, then $H: \operatorname{dom}(L) \subset X \rightarrow Y$ is a linear bijection with bounded inverse and $\left(J Q+K_{p}(I-Q)\right)(L+$ $\left.J^{-1} P\right)=\left(L+J^{-1} P\right)\left(J Q+K_{p}(I-Q)\right)=I$. From [12] we see that $K_{1}=H(K \cap \operatorname{dom}(L))$ is a cone in $Y$ and we have the following theorem.

Theorem 1.1 [12] $N(u)+J^{-1} P(u)=H(\bar{u})$, where $\bar{u}=P(u)+J Q N(u)+K_{p}(I-Q) N(u)$ and $\bar{u}$ is uniquely determined.

As a consequence of the above theorem, the author obtained the equivalence of the following two assertions:

(i) $P+J Q N+K_{p}(I-Q) N: K \cap \operatorname{dom}(L) \rightarrow K \cap \operatorname{dom}(L)$,

(ii) $N+J^{-1} P: K \cap \operatorname{dom}(L) \rightarrow K_{1}$.

Now we introduce the notion of lower and upper solutions.

Definition 1.2 [6] Let $K$ be a normal cone in a Banach space $X, u_{0} \leq v_{0}$, and $u_{0}, v_{0} \in$ $K \cap \operatorname{dom}(L)$ are said to be coupled lower and upper solutions of the equation $L u=N u$ if

$$
\left\{\begin{array}{l}
L u_{0} \leq N u_{0} \\
L v_{0} \geq N v_{0}
\end{array}\right.
$$

Theorem 1.3 [6] Let $L: \operatorname{dom}(L) \subset X \rightarrow Y$ be a Fredholm operator of index zero, $K$ be a normal cone in Banach space $X, u_{0}, v_{0} \in K \cap \operatorname{dom}(L), u_{0} \leq v_{0}$, and $N:\left[u_{0}, v_{0}\right] \rightarrow Y$ be $L$-compact and continuous. Suppose that the following conditions are satisfied:

$\left(\mathrm{C}_{1}\right) u_{0}$ and $v_{0}$ are coupled lower and upper solutions of the equation $\mathrm{Lu}=\mathrm{Nu}$.

$\left(C_{2}\right) N+J^{-1} P: K \cap \operatorname{dom}(L) \rightarrow K_{1}$ is an increasing operator.

Then the equation $L u=N u$ has a minimal solution $u^{*}$ and a maximal solution $v^{*}$ in $\left[u_{0}, v_{0}\right]$.

$$
\begin{gathered}
\text { Moreover, } u^{*}=\lim _{n \rightarrow \infty} u_{n} \text {, and } v^{*}=\lim _{n \rightarrow \infty} v_{n} \text {, where } \\
u_{n}=\left(L+J^{-1} P\right)^{-1}\left(N+J^{-1} P\right) u_{n-1}, \\
v_{n}=\left(L+J^{-1} P\right)^{-1}\left(N+J^{-1} P\right) v_{n-1}, \quad \text { for } n=1,2,3, \ldots, \\
u_{0} \leq u_{1} \leq u_{2} \leq \cdots \leq u_{n} \leq \cdots \leq v_{n} \leq \cdots \leq v_{2} \leq v_{1} \leq v_{0} .
\end{gathered}
$$

\section{Preliminaries}

Now, we introduce some notations, definitions and preliminary facts which will be used throughout this paper.

Definition 2.1 The Riemann-Liouville fractional integral operator of order $\alpha>0$ of a function $g$ is defined by

$$
I_{a^{+}}^{\alpha} g(t)=\frac{1}{\Gamma(\alpha)} \int_{a}^{t}(t-s)^{\alpha-1} g(s) d s,
$$

provided that the right side integral is pointwise defined on $(0,+\infty)$. 
Definition 2.2 The Caputo fractional derivative of order $\alpha>0$ of a continuous function $g$ is given by

$$
{ }^{c} D_{a^{+}}^{\alpha} g(t)=\frac{1}{\Gamma(n-\alpha)} \int_{a}^{t}(t-s)^{n-\alpha-1} g^{(n)}(s) d s,
$$

when $n$ is the smallest integer greater than or equal to $\alpha$, provided that the right side integral is pointwise defined on $(0,+\infty)$.

Lemma 2.3 For $\alpha>0, g \in C([0,1], \mathbb{R})$, the homogeneous fractional differential equation ${ }^{c} D_{a^{+}}^{\alpha} g(t)=0$ has a solution $g(t)=c_{0}+c_{1} t+c_{2} t^{2}+\cdots+c_{n-1} t^{n-1}$, where $c_{i} \in \mathbb{R}, i=0, \ldots, n-1$, here $n$ is the smallest integer greater than or equal to $\alpha$.

Let $X=Y=C[0,1]$ equipped with the norm $\|u\|=\sup _{t \in[0,1]}|u(t)|$ and $K=\{u \in X: u(t) \geq$ $0, t \in[0,1]\}$.

Define the operators $L$ and $N$, respectively, by $L: \operatorname{dom}(L) \subset X \rightarrow Y$

$$
L u(t)={ }^{c} D_{0^{+}}^{\alpha} u(t)
$$

$\operatorname{dom}(L)=\left\{u \in A C^{2}[0,1]:{ }^{c} D_{0^{+}}^{\alpha} u(t) \in C[0,1], u(0)=u^{\prime}(0)=0, u^{\prime \prime}(0)=2 u(1)\right\}$ and $N: X \rightarrow Y$

$$
N u(t)=f(t, u(t)), \quad \forall t \in[0,1]
$$

then the boundary value problem (P) can be written as $L u=N u, u \in K \cap \operatorname{dom}(L)$.

Lemma 2.4 We have

$$
\operatorname{Ker} L=\left\{u \in \operatorname{dom}(L): u(t)=c t^{2}, c \in \mathbb{R}, \forall t \in[0,1]\right\}
$$

and

$$
\operatorname{Im} L=\left\{y \in Y: \int_{0}^{1}(1-s)^{\alpha-1} y(s) d s=0\right\} .
$$

Proof By Lemma 2.3, the function $u(t)=c_{0}+c_{1} t+c_{2} t^{2}, c_{0}, c_{1}, c_{2} \in \mathbb{R}$ is the solution of $L u(t)=D_{0^{+}}^{\alpha} u(t)=0$. Taking into account the boundary conditions (1.2), we get

$$
\operatorname{Ker} L=\left\{u \in \operatorname{dom}(L): u(t)=c t^{2}, c \in \mathbb{R}, \forall t \in[0,1]\right\} .
$$

Let us show that

$$
\operatorname{Im} L=\left\{y \in Y: \int_{0}^{1}(1-s)^{\alpha-1} y(s) d s=0\right\} .
$$

For $y \in \operatorname{Im} L$, there exists $u \in \operatorname{dom}(L)$ such that $y=L u \in Y$. By Lemma 2.3, it follows that

$$
u(t)=\frac{1}{\Gamma(\alpha)} \int_{0}^{t}(t-s)^{\alpha-1} y(s) d s+c_{0}+c_{1} t+c_{2} t^{2} .
$$


It is easy to get

$$
\begin{aligned}
& u^{\prime}(t)=\frac{1}{\Gamma(\alpha-1)} \int_{0}^{t}(t-s)^{\alpha-2} y(s) d s+c_{1}+2 c_{2} t \\
& u^{\prime \prime}(t)=\frac{1}{\Gamma(\alpha-2)} \int_{0}^{t}(t-s)^{\alpha-3} y(s) d s+2 c_{2}
\end{aligned}
$$

then the boundary conditions (1.2) imply

$$
\int_{0}^{1}(1-s)^{\alpha-1} y(s) d s=0
$$

On the other hand, suppose that $y \in Y$ and satisfies $\int_{0}^{1}(1-s)^{\alpha-1} y(s) d s=0$. Let $u(t)=$ $I_{0^{+}}^{\alpha} y(t)+c t^{2}$, then $u \in \operatorname{dom}(L)$ and $D_{0^{+}}^{\alpha} u(t)=y(t)$. Thus, $y \in \operatorname{Im} L$.

Now, define the operators $P: X \rightarrow X$ by

$$
P u(t)=\frac{1}{2} \alpha(\alpha+1)(\alpha+2) t^{2} \int_{0}^{1}(1-s)^{\alpha-1} u(s) d s
$$

and $Q: Y \rightarrow Y$ by

$$
Q y(t)=\alpha \int_{0}^{1}(1-s)^{\alpha-1} y(s) d s, \quad \forall t \in[0,1] .
$$

It is easy to see that the operators $P$ and $Q$ are both projectors. In fact, for $t \in[0,1]$,

$$
\begin{aligned}
P^{2} u(t) & =P(P u)(t) \\
& =\frac{1}{2} \alpha(\alpha+1)(\alpha+2) t^{2} \int_{0}^{1}(1-s)^{\alpha-1}(P u)(s) d s \\
& =\frac{1}{4} \alpha^{2}(\alpha+1)^{2}(\alpha+2)^{2} t^{2} \int_{0}^{1}(1-s)^{\alpha-1} u(s) d s \int_{0}^{1}(1-s)^{\alpha-1} s^{2} d s \\
& =\frac{1}{2} \alpha(\alpha+1)(\alpha+2) t^{2} \int_{0}^{1}(1-s)^{\alpha-1} u(s) d s=P u(t) .
\end{aligned}
$$

Similarly we show that $Q$ is a projector. Obviously, $\operatorname{Im} P=\operatorname{Ker} L$ and $\operatorname{Ker} Q=\operatorname{Im} L$.

Lemma 2.5 The operator $L: \operatorname{dom}(L) \subset X \rightarrow Y$ is a Fredholm operator of index zero, and its inverse $K_{p}: \operatorname{Im} L \rightarrow \operatorname{dom}(L) \cap \operatorname{Ker} P$ is given by

$$
K_{p} y(t)=\int_{0}^{1} k(t, s) y(s) d s, \quad \forall t \in[0,1]
$$

where

$$
k(t, s)= \begin{cases}\frac{(t-s)^{\alpha-1}}{\Gamma(\alpha)}-\alpha(\alpha+1)(\alpha+2) t^{2} \frac{\Gamma(\alpha)}{2 \Gamma(2 \alpha)}(1-s)^{2 \alpha-1}, & 0 \leq s \leq t \leq 1 \\ -\alpha(\alpha+1)(\alpha+2) t^{2} \frac{\Gamma(\alpha)}{2 \Gamma(2 \alpha)}(1-s)^{2 \alpha-1}, & 0 \leq t \leq s \leq 1\end{cases}
$$


Proof From $u=(u-P u)+P u$ it follows that $X=\operatorname{Ker} P+\operatorname{Ker} L$. By simple calculation, we obtain $\operatorname{Ker} L \cap \operatorname{Ker} P=\{0\}$, then $X=\operatorname{Ker} L \oplus \operatorname{Ker} P$. By the same idea we prove that $Y=$ $\operatorname{Im} L \oplus \operatorname{Im} Q$. Thus

$$
\operatorname{dim} \operatorname{Ker} L=\operatorname{dim} \operatorname{Im} Q=\operatorname{codim} \operatorname{Im} L=1 .
$$

This means that $L$ is a Fredholm operator of index zero.

Let us find the expression of $K_{p}: \operatorname{Im} L \rightarrow \operatorname{dom}(L) \cap \operatorname{Ker} P$. Let $u \in \operatorname{dom}(L) \cap \operatorname{Ker} P$, then $y(t)={ }^{c} D_{0^{+}}^{\alpha} u(t) \in \operatorname{Im} L$ and

$$
K_{P} y(t)=u(t)=I_{0^{+}}^{\alpha} y(t)+C t^{2}=\frac{1}{\Gamma(\alpha)} \int_{0}^{t}(t-s)^{\alpha-1} y(s) d s+C t^{2} .
$$

Since $u \in \operatorname{dom}(L) \cap \operatorname{Ker} P$,

$$
\begin{aligned}
0 & =\int_{0}^{1}(1-t)^{\alpha-1} u(t) d t \\
& =\frac{1}{\Gamma(\alpha)} \int_{0}^{1}(1-t)^{\alpha-1} \int_{0}^{t}(t-s)^{\alpha-1} y(s) d s d t+C \int_{0}^{1} t^{2}(1-t)^{\alpha-1} d t \\
& =\frac{1}{\Gamma(\alpha)} \int_{0}^{1} y(s) \int_{s}^{1}(1-t)^{\alpha-1}(t-s)^{\alpha-1} d t d s+\frac{2 C}{\alpha(\alpha+1)(\alpha+2)}
\end{aligned}
$$

thus

$$
\begin{aligned}
C & =-\frac{\alpha(\alpha+1)(\alpha+2)}{2 \Gamma(\alpha)} \int_{0}^{1} y(s) \int_{s}^{1}(1-t)^{\alpha-1}(t-s)^{\alpha-1} d t d s, \\
& =-\alpha(\alpha+1)(\alpha+2) \frac{\Gamma^{2}(\alpha)}{2 \Gamma(2 \alpha)} \int_{0}^{1}(1-s)^{2 \alpha-1} y(s) d s .
\end{aligned}
$$

Substituting $C$ by its value in (2.2) we get

$$
\begin{aligned}
\left(K_{P} y\right)(t)= & \frac{1}{\Gamma(\alpha)} \int_{0}^{t}(t-s)^{\alpha-1} y(s) d s \\
& -\alpha(\alpha+1)(\alpha+2) t^{2} \frac{\Gamma(\alpha)}{2 \Gamma(2 \alpha)} \int_{0}^{1}(1-s)^{2 \alpha-1} y(s) d s \\
= & \int_{0}^{1} k(t, s) y(s) d s,
\end{aligned}
$$

where $k(t, s)$ is given by $(2.1)$.

\section{Main result}

Define the isomorphism $J: \operatorname{Im} Q \rightarrow \operatorname{Ker} L$ by $J(c)=\frac{1}{2}(\alpha+1)(\alpha+2) c t^{2}$. We have the following result.

Lemma 3.1 We have

$$
\left(J Q N+K_{p}(I-Q) N\right) u(t)=\int_{0}^{1} G(t, s) f(s, u(s)) d s,
$$


where

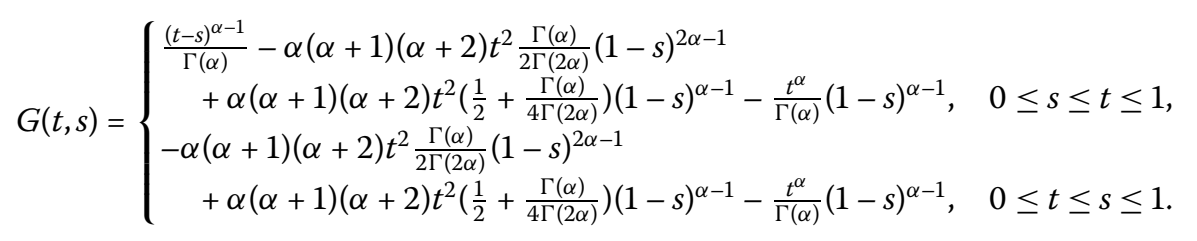

$G$ is continuous and nonnegative on $[0,1] \times[0,1]$.

Proof We have

$$
\begin{aligned}
& Q N u(t)=\alpha \int_{0}^{1}(1-s)^{\alpha-1} f(s, u(s)) d s, \\
& \begin{aligned}
K_{p}(I-Q) N u(t)= & \int_{0}^{1} k(t, s) f(s, u(s)) d s \\
& \quad-\alpha\left(\int_{0}^{1}(1-s)^{\alpha-1} f(s, u(s)) d s\right)\left(\int_{0}^{1} k(t, s) d s\right) .
\end{aligned}
\end{aligned}
$$

Then

$$
\begin{aligned}
(J Q N & \left.+K_{p}(I-Q) N\right) u(t) \\
= & \frac{\alpha(\alpha+1)(\alpha+2) t^{2}}{2} \int_{0}^{1}(1-s)^{\alpha-1} f(s, u(s)) d s \\
& +\int_{0}^{1} k(t, s) f(s, u(s)) d s-\alpha\left(\int_{0}^{1}(1-s)^{\alpha-1} f(s, u(s)) d s\right)\left(\int_{0}^{1} k(t, s) d s\right) \\
= & \frac{\alpha(\alpha+1)(\alpha+2) t^{2}}{2} \int_{0}^{1}(1-s)^{\alpha-1} f(s, u(s)) d s \\
& +\frac{1}{\Gamma(\alpha)} \int_{0}^{t}(t-s)^{\alpha-1} f(s, u(s)) d s \\
& -\alpha(\alpha+1)(\alpha+2) t^{2} \frac{\Gamma(\alpha)}{2 \Gamma(2 \alpha)} \int_{0}^{1}(1-s)^{2 \alpha-1} f(s, u(s)) d s \\
& +\left(-\frac{t^{\alpha}}{\Gamma(\alpha)}+\alpha(\alpha+1)(\alpha+2) t^{2} \frac{\Gamma(\alpha)}{4 \Gamma(2 \alpha)}\right)\left(\int_{0}^{1}(1-s)^{\alpha-1} f(s, u(s)) d s\right) .
\end{aligned}
$$

It is easy to see that $G$ is continuous according to both variables $s, t \in[0,1]$. Let $t \leq s \leq 1$, then

$$
\begin{aligned}
G(t, s)= & -\alpha(\alpha+1)(\alpha+2) t^{2} \frac{\Gamma(\alpha)}{2 \Gamma(2 \alpha)}(1-s)^{2 \alpha-1} \\
& +\alpha(\alpha+1)(\alpha+2) t^{2}\left(\frac{1}{2}+\frac{\Gamma(\alpha)}{4 \Gamma(2 \alpha)}\right)(1-s)^{\alpha-1}-\frac{t^{\alpha}}{\Gamma(\alpha)}(1-s)^{\alpha-1} \\
\geq & \left(-\alpha(\alpha+1)(\alpha+2) t^{2} \frac{\Gamma(\alpha)}{4 \Gamma(2 \alpha)}-\frac{t^{2}}{\Gamma(\alpha)}+\frac{1}{2} \alpha(\alpha+1)(\alpha+2) t^{2}\right)(1-s)^{\alpha-1} \\
\geq & \left(-\alpha(\alpha+1)(\alpha+2) \frac{\Gamma(\alpha)}{4 \Gamma(2 \alpha)}-\frac{1}{\Gamma(\alpha)}+\frac{1}{2} \alpha(\alpha+1)(\alpha+2)\right) t^{2}(1-s)^{\alpha-1} \\
\geq & 6 t^{2}(1-s)^{\alpha-1} \geq 0 .
\end{aligned}
$$


Similarly we get, for $0 \leq s \leq t \leq 1$,

$$
G(t, s) \geq \frac{(t-s)^{\alpha-1}}{\Gamma(\alpha)}+6 t^{2}(1-s)^{\alpha-1} \geq 0
$$

The proof is complete.

Lemma 3.2 The operator $N$ is L-compact and continuous on $\bar{\Omega}$, where $\Omega$ is any open bounded subset of $K \cap \operatorname{dom}(L)$.

Proof We have to prove that $Q N(\bar{\Omega})$ and $K_{p}(I-Q)(\bar{\Omega})$ are both compact. Let $u \in \bar{\Omega}$ and $M=\max (f(s, u(s)), 0 \leq s \leq 1, u \in \bar{\Omega})$, remarking that $|k(t, s)| \leq 21$, we easily get

$$
\begin{aligned}
\left|K_{p}(I-Q) N u(t)\right| \leq & \int_{0}^{1} f(s, u(s))|k(t, s)| d s \\
& +\alpha\left(\int_{0}^{1}(1-s)^{\alpha-1} f(s, u(s)) d s\right)\left(\int_{0}^{1}|k(t, s)| d s\right) \leq 42 M,
\end{aligned}
$$

thus $\left\|K_{p}(I-Q) N u\right\| \leq 42 M$, so $K_{p}(I-Q) N$ is uniformly bounded on $\bar{\Omega}$.

Let $0 \leq t_{1}<t_{2} \leq 1$, then

$$
\begin{aligned}
\left|K_{p}(I-Q) N u\left(t_{2}\right)-K_{p}(I-Q) N u\left(t_{1}\right)\right| \\
\leq \frac{1}{\Gamma(\alpha)} \int_{0}^{t_{1}}\left(\left(t_{2}-s\right)^{\alpha-1}-\left(t_{1}-s\right)^{\alpha-1}\right) f(s, u(s)) d s \\
\quad+\frac{1}{\Gamma(\alpha)} \int_{t_{1}}^{t_{2}}\left(t_{2}-s\right)^{\alpha-1} f(s, u(s)) d s \\
\quad+\alpha(\alpha+1)(\alpha+2)\left(t_{2}^{2}-t_{1}^{2}\right) \frac{\Gamma(\alpha)}{2 \Gamma(2 \alpha)} \int_{0}^{1}(1-s)^{2 \alpha-1} f(s, u(s)) d s \\
\quad+\left(\int_{0}^{1}(1-s)^{\alpha-1} f(s, u(s)) d s\right)\left(\left(t_{2}^{\alpha}-t_{1}^{\alpha}\right)+(\alpha+1)(\alpha+2)\left(t_{2}^{2}-t_{1}^{2}\right) \frac{\Gamma(\alpha)}{2 \Gamma(2 \alpha)}\right) \\
\leq \frac{M}{\Gamma(\alpha)}\left[\alpha\left(t_{2}-t_{1}\right)+\frac{\Gamma^{2}(\alpha)}{2 \Gamma(2 \alpha)}(\alpha+1)^{2}(\alpha+2)\left(t_{2}^{2}-t_{1}^{2}\right)+\left(t_{2}^{\alpha}-t_{1}^{\alpha}\right)\right] .
\end{aligned}
$$

As $t_{1} \rightarrow t_{2}$, the right-hand side of the above inequality tends to 0 , consequently $K_{p}(I-$ $Q)(\bar{\Omega})$ is equicontinuous. By means of the Arzela-Ascoli theorem we conclude that $K_{p}(I-$ $Q)(\bar{\Omega})$ is compact. Similarly we prove that $Q N(\bar{\Omega})$ is compact.

Theorem 3.3 Assume that:

$\left(\mathrm{H}_{1}\right)$ There exist $u_{0}, v_{0} \in K \cap \operatorname{dom}(L)$ such that $u_{0} \leq v_{0}$ and

$$
\begin{cases}{ }^{c} D_{0^{+}}^{\alpha} u_{0}(t) \leq f\left(t, u_{0}(t)\right), & \forall t \in[0,1] \\ { }^{c} D_{0^{+}}^{\alpha} v_{0}(t) \geq f\left(t, v_{0}(t)\right), & \forall t \in[0,1]\end{cases}
$$

$\left(\mathrm{H}_{2}\right)$ For any $x, y \in K \cap \operatorname{dom}(L), u_{0}(t) \leq y(t) \leq x(t) \leq v_{0}(t), \forall t \in[0,1]$, the function $f$ satisfies

$$
f(t, x(t))-f(t, y(t)) \geq-\alpha\left(\int_{0}^{1}(1-t)^{\alpha-1} x(t) d t-\int_{0}^{1}(1-t)^{\alpha-1} y(t) d t\right) .
$$


Then the boundary value problem (P) has a minimal solution $u^{*}$ and a maximal solution $v^{*}$ in $\left[u_{0}, v_{0}\right]$.

Proof We will prove that all conditions of Theorem 1.3 are satisfied. From the proof of Lemma 2.5, we know that $L$ is a Fredholm operator of index zero. In view of condition $\left(\mathrm{H}_{1}\right)$, we get $L u_{0} \leq N u_{0}$ and $L v_{0} \geq N v_{0}$, so condition $\left(\mathrm{C}_{1}\right)$ of Theorem 1.3 holds. For $u \in K$, we have

$$
\begin{aligned}
(P & \left.+J Q N+K_{p}(I-Q) N\right) u(t) \\
& =\frac{1}{2} \alpha(\alpha+1)(\alpha+2) t^{2} \int_{0}^{1}(1-s)^{\alpha-1} u(s)+\int_{0}^{1} G(t, s) f(s, u(s)) d s .
\end{aligned}
$$

Since $G(t, s)$ is continuous and nonnegative for $t, s \in[0,1],\left(P+J Q N+K_{p}(I-Q) N\right)(K) \subset K$. By virtue of the equivalence assertions, we conclude that $N+J^{-1} P: K \cap \operatorname{dom}(L) \rightarrow K_{1}$. Condition $\left(\mathrm{H}_{2}\right)$ implies that $N+J^{-1} P: K \cap \operatorname{dom}(L) \rightarrow K_{1}$ is a monotone increasing operator, in fact for $x, y \in K \cap \operatorname{dom}(L), y(t) \leq x(t), \forall t \in[0,1]$, we have

$$
\begin{aligned}
(N & \left.+J^{-1} P\right) x(t)-\left(N+J^{-1} P\right) y(t) \\
& =f(t, x(t))-f(t, y(t))+\alpha\left(\int_{0}^{1}(1-t)^{\alpha-1} x(t) d t-\int_{0}^{1}(1-t)^{\alpha-1} y(t) d t\right) \geq 0,
\end{aligned}
$$

so condition $\left(\mathrm{C}_{2}\right)$ is satisfied. Finally, we conclude by Theorem 1.3 that the equation $L u=$ $N u$ has a minimal solution $u^{*}$ and a maximal solution $v^{*}$ in $\left[u_{0}, v_{0}\right]$, where $u^{*}=\lim _{n \rightarrow \infty} u_{n}$ and $v^{*}=\lim _{n \rightarrow \infty} v_{n}$, uniformly according to $t$, the sequences $u_{n}$ and $v_{n}$ are defined by

$$
\begin{aligned}
u_{n}= & \left(L+J^{-1} P\right)^{-1}\left(N+J^{-1} P\right) u_{n-1} \\
= & \left(J Q+K_{p}(I-Q)\right)\left(N+J^{-1} P\right) u_{n-1} \\
= & \left(J Q+K_{p}(I-Q)\right)\left(f\left(s, u_{n-1}(s)\right)+\alpha \int_{0}^{1}(1-s)^{\alpha-1} u_{n-1}(s) d s\right) \\
= & \frac{1}{\Gamma(\alpha)} \int_{0}^{t}(t-s)^{\alpha-1} f\left(s, u_{n-1}(s)\right) d s \\
& +\alpha(\alpha+1)(\alpha+2) t^{2} \frac{\Gamma(\alpha)}{4 \Gamma(2 \alpha)} \int_{0}^{1}(1-s)^{\alpha-1} f\left(s, u_{n-1}(s)\right) d s \\
& -\frac{t^{\alpha}}{\Gamma(\alpha)} \int_{0}^{1}(1-s)^{\alpha-1} f\left(s, u_{n-1}(s)\right) d s \\
& -\alpha(\alpha+1)(\alpha+2) \frac{t^{2} \Gamma(\alpha)}{2 \Gamma(2 \alpha)} \int_{0}^{1}(1-s)^{2 \alpha-1} f\left(s, u_{n-1}(s)\right) d s \\
& +\frac{1}{2} \alpha(\alpha+1)(\alpha+2) t^{2} \int_{0}^{1}(1-s)^{\alpha-1} u_{n-1}(s) d s,
\end{aligned}
$$

similarly we get the expression of $v_{n}$, moreover, we have

$$
u_{0} \leq u_{1} \leq u_{2} \leq \cdots \leq u_{n} \leq \cdots \leq v_{n} \leq \cdots \leq v_{2} \leq v_{1} \leq v_{0} .
$$


Example 3.4 Let us consider the following fractional boundary value problem:

$$
\begin{cases}{ }^{c} D_{0^{+}}^{\frac{5}{2}} u(t)=t^{2}+\frac{u}{u+1}, & 0<t<1 \\ u(0)=u^{\prime}(0)=0, & u^{\prime \prime}(0)=2 u(1) .\end{cases}
$$

We can choose

$$
u_{0}(t)=\frac{1}{\Gamma\left(\frac{5}{2}\right)} \int_{0}^{t}(t-s)^{\frac{3}{2}} s^{2} d s \leq \frac{1}{\Gamma\left(\frac{5}{2}\right)} \int_{0}^{t}(t-s)^{\frac{3}{2}}(s+1)^{2} d s=v_{0}(t)
$$

then

$$
\begin{aligned}
& { }^{c} D_{0^{+}}^{\frac{5}{2}} u_{0}(t)=t^{2} \leq(t+1)^{2}={ }^{c} D_{0^{+}}^{\frac{5}{2}} \nu_{0}(t), \\
& { }^{c} D_{0^{+}}^{\frac{5}{2}} u_{0}(t) \leq f\left(t, u_{0}(t)\right), \quad{ }^{c} D_{0^{+}}^{\frac{5}{2}} \nu_{0}(t) \geq f\left(t, v_{0}(t)\right), \quad \forall t \in[0,1] .
\end{aligned}
$$

For any $x, y \in K \cap \operatorname{dom}(L)$, we have

$$
\left(t^{2}+\frac{x}{x+1}\right)-\left(t^{2}+\frac{y}{y+1}\right) \geq-\frac{5}{2}\left(\int_{0}^{1}(1-t)^{\alpha-1} x(t) d t-\int_{0}^{1}(1-t)^{\alpha-1} y(t) d t\right),
$$

where $u_{0}(t) \leq y(t) \leq x(t) \leq v_{0}(t), \forall t \in[0,1]$. Then, by Theorem 3.3, the boundary value problem (3.1) has a minimal solution $u^{*}$ and a maximal solution $v^{*}$ in $\left[u_{0}, v_{0}\right]$.

\section{Competing interests}

The authors declare that they have no competing interests.

Authors' contributions

All authors contributed in writing this paper. All authors read and approved the final manuscript.

\section{Author details}

${ }^{1}$ Laboratory of Advanced Materials, Faculty of Sciences, Badji Mokhtar-Annaba University, P.O. Box 12, Annaba, 23000 , Algeria. ${ }^{2}$ Department of Mathematics, University 08 Mai 45-Guelma, P.O. Box 401, Guelma, 24000, Algeria.

\section{Acknowledgements}

The authors thank the referee for his (her) helpful comments on the manuscript.

Received: 23 April 2015 Accepted: 6 October 2015 Published online: 14 October 2015

\section{References}

1. Bai, C, Fang, J: Existence of positive solutions for boundary value problems at resonance. J. Math. Anal. Appl. 291, 538-549 (2004)

2. Chen, $Y$, Tang, $X$ : Positive solutions of fractional differential equations at resonance on the half-line. Bound. Value Probl. 2012, 64 (2012)

3. Han, X: Positive solutions for a three-point boundary value problem at resonance. J. Math. Anal. Appl. 36, 556-568 (2007)

4. Infante, G, Zima, M: Positive solutions of multi-point boundary value problems at resonance. Nonlinear Anal. 69 2458-2465 (2008)

5. Kosmatov, N: Multi-point boundary value problems on an unbounded domain at resonance. Nonlinear Anal. 68 2158-2171 (2008)

6. Liang, SQ, Mu, L: Multiplicity of positive solutions for singular three-point boundary value problem at resonance. Nonlinear Anal. 71, 2497-2505 (2009)

7. Yang, A, Wang, $\mathrm{H}$ : Positive solutions of two-point boundary value problems of nonlinear fractional differential equation at resonance. Electron. J. Qual. Theory Differ. Equ. 2011, 71 (2011)

8. Zhang, HE, Sun, J-P: Positive solutions of third-order nonlocal boundary value problems at resonance. Bound. Value Probl. 2012, 102 (2012)

9. Qu, H, Liu, X: Existence of nonnegative solutions for a fractional $m$-point boundary value problem at resonance. Bound. Value Probl. 2013, 127 (2013)

10. Wang, F, Cui, YJ, Zhang, F: Existence of nonnegative solutions for second order $m$-point boundary value problems at resonance. Appl. Math. Comput. 217, 4849-4855 (2011)

11. Gaines, RE, Mawhin, JL: Coincidence Degree and Nonlinear Differential Equation. Springer, Berlin (1977)

12. Cremins, CT: A fixed-point index and existence theorems for semilinear equations in cones. Nonlinear Anal. 42 , 789-806 (2001) 